\section{SARS-COV Sampling from 3 Portals}

To the Editor: Wang et al. detected severe acute respiratory syndrome-associated coronavirus (SARS$\mathrm{CoV}$ ) from throat wash and saliva specimens and suggested that these specimens have advantages over other specimens, including ease of procurement and safety for medical personnel (1). The virus has been detected with variable success from nasopharyngeal aspirates, nose and throat swabs, and tears $(2,3)$. Advocates of all of these sampling methods emphasize the need for early diagnosis of SARS. The probability for nosocomial transmission to healthcare workers when they obtain specimens from patients has not been adequately addressed. In a study of outbreak control for SARS, Chowell et al. suggest “ $\ldots$ the strong sensitivity of $R_{0}$ to the transmission rate $\beta$ indicates that efforts in finding intervention strategies that manage to systematically lower the contact rate of persons of all age groups promise an effective means for lowering $R_{0}$ " (4).

An important component of a comprehensive strategy to lower the contact rate is improving the safety measures recommended for clinical specimen collection by healthcare workers. Recognizing the importance of obtaining multiple specimens and the difficulties associated with obtaining samples from the 3 usual portals of entry, we devised and tested a novel method of specimen collection, conjunctiva-upper respiratory tract irrigation (5). We coupled our specimen collection method with detailed written instructions to enable the patients themselves to perform the entire procedures. Almost all other specimen collection methods require assistance from healthcare workers or have other limitations, such as inabil- ity to sample all 3 portals. The method is not perfect because some persons have difficulty performing the procedure; however, self-instillation of the irrigation into the nostrils, with or without the addition of a throat wash or saliva, is likely to improve the success rate. The data supplied by Loon et al. (3) and Wang et al. (1) confirm that collecting specimens by a method that involves minimal contact between a possible source of infection and susceptible persons is desirable.

The author has shares of a company that owns a patent-pending conjunctivaupper respiratory tract irrigation system.

\section{Tommy R. Tong*}

*Princess Margaret Hospital, Hong Kong, Special Administrative Region, China

\section{References}

1. Wang WK, Chen SY, Liu IJ, Chen YC, Chen HL, Yang CF, et al. Detection of SARS-associated coronavirus in throat wash and saliva in early diagnosis. Emerg Infect Dis. 2004;10:1213-9.

2. Chan PK, To WK, Ng KC, Lam RK, Ng TK, Chan RC, et al. Laboratory diagnosis of SARS. Emerg Infect Dis. 2004;10:825-31.

3. Loon SC, Teoh SC, Oon LL, Se-Thoe SY, Ling AE, Leo YS, et al. The severe acute respiratory syndrome coronavirus in tears. Br J Ophthalmol. 2004;88:861-3.

4. Chowell G, Castillo-Chavez C, Fenimore PW, Kribs-Zaleta CM, Arriola L, Hyman JM. Model parameters and outbreak control for SARS. Emerg Infect Dis. 2004;10:1258-63.

5. Tong TR, Lam BH, Ng TK, Lai ST, Tong MK, Chau TN. Conjunctiva-upper respiratory tract irrigation for early diagnosis of severe acute respiratory syndrome. J Clin Microbiol. 2003;41:5352.

Address for correspondence: Tommy R Tong, Department of Pathology, Room P-725, Block P, 7/F, Princess Margaret Hospital, Kowloon, Hong Kong; fax: +852-29903377; email: tommytong@yahoo.com

\section{Occupational Health Response to SARS}

To the Editor: Severe acute respiratory syndrome (SARS), an occupational disease risk for healthcare workers, warrants an occupational health response, as clearly described by Esswein et al. (1). Occupational health professionals played a role in the assessment of healthcare facilities in Taiwan and many other countries. For example, occupational health professionals were invited to perform audits in at least 2 hospitals in Singapore during the height of the crisis, (2) and to conduct follow-up discussions with the hospital management. In addition to assessment of the industrial hygiene aspects, which included evaluating the ventilation modifications needed for effective infection control, temperature and humidity were significant factors affecting the use of protective gear in a tropical country like Singapore. The occupational health audits included site inspections and reviews of work processes of those areas where actual transmission of SARS had occurred and where triage of febrile patients was taking place. Other issues identified as requiring urgent attention were providing sufficient rest, shower, and changing facilities for staff, monitoring staff sickness absenteeism, and proactively managing staff mental health. Occupational health physicians subsequently served on hospital SARS debriefing committees that reviewed institutional shortcomings and recommended new measures for future outbreaks. An occupational health service unit headed by a trained occupational health physician was formed in 1 hospital.

Other occupational groups, as well as healthcare workers, are also at potential risk. These groups may include the following: 1) food handlers, defined as persons who handle, 
kill, or sell food animals, and persons who prepare and serve food. (More than one third of the cases in China with onset of SARS before February 1, 2003, were in food handlers [3].); 2) public transportation workers and airline crew (4); and 3) laboratory workers handling samples or items contaminated with SARS-associated coronavirus (5). In Singapore, 2 taxi drivers were infected after ferrying SARS patients to healthcare facilities, and 1 Singapore Airlines cabin attendant came down with the infection after a flight with infected passengers on board. Occupationally acquired SARS infections have been documented in Singapore, Taiwan, and Beijing. Clearly, occupational health responses are needed in these occupational settings.

The recognition of SARS as an occupational disease has broader implications. Depending on country legislation, persons who contract SARS while performing their work may be eligible for worker's compensation. Employers would be obligated to provide a safe and healthy workplace for their employees.

\section{David Koh, ${ }^{*}$ Meng-Kin Lim, ${ }^{*}$ Choon-Nam Ong,* and Sin-Eng Chia*}

*National University of Singapore, Singapore

\section{References}

1. Esswein EJ, Kiefer M, Wallingford K, Burr G, Lee LJH, Wang JD, et al. Environmental and occupational health response to SARS, Taiwan, 2003. Emerg Infect Dis. 2004;10:1187-94.

2. Koh D, Lim MK, Chia SE. SARS: health care work can be hazardous to health. Occup Med. 2003;53:241-3.

3. World Health Organization. Consensus document on the epidemiology of severe acute respiratory syndrome (SARS). 17 Oct 2003. Geneva: The Organization; 2003.

4. Olsen SJ, Chang HL, Cheung TY, Tang AF, Fisk TL, Ooi SP, et al. Transmission of the severe acute respiratory syndrome on aircraft. N Engl J Med. 2003;349:2416-22.
5. Normile D. Severe acute respiratory syndrome: lab accidents prompt calls for new containment program. Science. 2004;304: 1223-5.

Address for correspondence: David Koh, Department of Community, Occupational and Family Medicine, Faculty of Medicine (MD3), National University of Singapore, 16 Medical Drive, Singapore 117597; fax: 65-6-7791489; email: cofkohd@nus.edu.sg

\section{Tracing SARS- Coronavirus} Variant with Large
Genomic Deletion

To the Editor: Severe acute respiratory syndrome (SARS) has been a global public health issue (1). We completed a study on the evolutionary path of the SARS-associated coronavirus (SARS-CoV) during the 2002-2003 epidemic (2). Most human SARS-CoV strains, as exemplified by the Tor2 sequence (GenBank accession no. AY274119) (3), are characterized by the deletion of a 29-nucleotide (nt) segment upstream of the nucleocapsid $(\mathrm{N})$ gene domain when compared with the viral strains isolated from the earliest human SARS patients (2) or from nonhuman mammalian hosts (4). Towards the end of the epidemic, a variant of the SARSCoV with a deletion of 386 nt flanking the 29-nt site was first demonstrated by complete genomic sequencing in 2 patients in Hong Kong (GenBank accession nos. AY394999, AY395000, AY395001, AY395002) (2). The 386nt deleted segment corresponds to the genomic region spanning residues 27719 to 28104 of the Tor2 sequence (3). The deletion results in the disruption of a putative open reading frame, orf 9 , while eliminating orfs 10 and 11. This deletion variant was first isolated from 2 SARS patients with disease onset in mid-May 2003. Patient A was a 41-year-old female phlebotomist working in North District Hospital, New Territories East Cluster, Hong Kong. Patient B was a 98-year-old woman admitted to ward X of North District Hospital (2).

With this finding late in the epidemic, we studied the prevalence of this SARS-CoV variant to determine its origin. Twenty-one SARS patients with disease onset dates from midApril were identified. All cases had been confirmed by positive reverse transcription-polymerase chain reaction (RT-PCR) detection of SARSCoV RNA in clinical specimens or seroconversion. These patients had been admitted with SARS to 4 different hospitals in Hong Kong, including North District Hospital and hospitals $\mathrm{A}$ and $\mathrm{B}$, which were located in the same geographic cluster as North District Hospital, as well as hospital C, which was geographically distant from the other 3 hospitals. Clinical specimens were retrieved, and RTPCR was performed to specifically amplify a genomic segment of SARS$\mathrm{CoV}$ encompassing the deletion site. Specimens with shortened PCR fragments were sequenced to determine the location and precise extent of the deletion.

RT-PCR products were not observed in 2 specimens. Gel electrophoresis of the RT-PCR products for each of the remaining 19 specimens showed a single genomic fragment; 13 of these fragments were shortened. Direct sequencing of the short amplicons showed a deletion of 386 nt identical to that isolated from patients $\mathrm{A}$ and $\mathrm{B}$. The patients' histories were reviewed. Patients $A, B$, and the 13 patients appeared to be epidemiologically related. The epidemiologic relationships and clinical details of the 15 cases are illustrated in the Figure. Most of the cases were part of a documented outbreak at North District Hospital traceable to an 